\title{
LAS LEMBRANÇAS PARA JAPÓN: OTRO ESCRITO INÉDITO DEL JESUITA GIL DE LA MATA
}

\author{
LEMBRANÇAS PARA JAPÓN: ANOTHER UNPUBLISHED \\ WORK WRITTEN BY THE JESUIT GIL DE LA MATA
}

\author{
Giuseppe Marino \\ The University of Tokyo. Tokyo, Japón \\ g.marino1982@gmail.com
}

\begin{abstract}
Resumen: La historia del primer intento de cristianización de Japón está aún por escribirse. El estudio de la teología moral misional, al igual que la investigación de los problemas éticos, podrían aportar nueva información sobre el primer contacto del Cristianismo con las culturas paganas. Los numerosos documentos, todavía poco conocidos, proponen soluciones a las cuestiones que afloraron en tierras de misión. Este estudio tiene como objetivo el rescate de un ulterior manuscrito inédito presente en el Archivo de la Compañía de Jesús en Roma (ARSJ). Las Lembranças para Japón, escrita de puño y letra por el misionero logroñés Gil de la Mata, son unas disposiciones en castellano del padre General, Claudio Aquaviva, contra la adaptación excesiva a las "catangues" de Japón promulgada por Alessandro Valignano. En este manuscrito residen las órdenes dirigidas al padre Visitador para preservar los principios de la Compañía de Jesús y contra el proceso de inculturación adoptado en aquel momento.
\end{abstract}

Palabras clave: Jesuitas, memorial, Claudio Aquaviva, Japón, Alessandro Valignano.

\begin{abstract}
The history of the first attempt to Christianize Japan has yet to be written. The study of moral theology's mission, as well as research into ethical problems, may provide new information about the first contact of Christianity with the pagan cultures. The numerous documents, still little known, propose solutions to the issues that crop up in the mission lands. This article aims to retrieve a subsequent unpublished manuscript found in the Archive of the Society of Jesus in Rome (ARSJ). The Lembranças of Japan, handwritten by the missionary Logroño Gil de la Mata, specifically consists of regulations or provisions in Spanish of the Padre General of the Jesuits Claudio Aquaviva against excessive adaptation to the "catangues" of Japan
\end{abstract}


promulgated by Alessandro Valignano. In this manuscript reside the orders directed to the Father Visitor to preserve the principles and foundations of the Society of Jesus and against the process of inculturation assumed at that time.

Keywords: Jesuits, memorial, Claudio Aquaviva, Japan, Alessandro Valignano.

Recibido: 02.06.2014. Aceptado:12.12.2014.

\section{Estado de la cuestión}

L PRESENTE arTículo quiere ser otra contribución, y un ulterior prose-
guimiento, a las investigaciones realizadas por el jesuita Jesús López Gay en su libro El Matrimonio de los japoneses (1964, pp. 79-130). A raíz de sus estudios sobre los problemas morales que surgieron en tierras de misiones, en particular durante el intento de cristianización del Japón en el siglo XVI, se ha investigado sobre una interesante problemática relativa al catecumenado en la Tierra del Sol Naciente: la asimilación de la Compañía de Jesús a la secta budista zen. Este dilema se engendró a partir del proceso de evangelización de las tierras niponas, exactamente cuando la joven Iglesia japonesa acababa de brotar y la incógnita central de los jesuitas parecía ser la adaptación a las costumbres de la vida del país de llegada. ¿Qué había que hacer?, ¿adaptarse o mantener vivos los principios y las reglas de la Institución? Por otro lado, existía otra controversia que concernía a la conversión de los japoneses: ¿qué era más oportuno?, ¿capturar la atención de los "gentiles" -involucrándose por medio de estima, simpatía y empatía, renunciando a la forma de vida occidental? o, ¿desistir instaurando una cristianización más puramente romano-ibérica sin bajar a compromisos inútiles y funestos para la integridad de la Compañía? Aproximadamente, todo empezó cuando el padre Visitador de la Compañía, Alessandro Valignano, se dio cuenta, durante su primera visita a Japón en 1579, de la enorme distancia entre los cristianos y la misma Iglesia. El jesuita italiano, siguiendo las enseñanzas de Francisco Javier, quiso llevar a cabo un tipo de catecumenado cuyo principio básico fuese principalmente la manifestación cultural. En concreto, impulsó a los misioneros para que profundizasen el conocimiento de la cultura nipona. Para empezar, decidió revocar las duras decisiones de Francisco Cabral, Provincial de la Compañía durante los años 1533-1609. La enculturación promulgada por Valignano, clave de la evan- 
gelización de Japón, consistió también en la adaptación del arte cristiano al conjunto de tradiciones culturales "del país de llegada", el cual los jesuitas se aproximaban a cristianizar (García Gutiérrez, 2012, p. 1473). Asimismo, una población tan diferente como la nipona, pero a la vez no indígena y con cierto grado de civilización, tenía que recibir una evangelización de forma igualmente distinta, a través de "su propia razón", sin intentar por la fuerza someter a los japoneses a la ley de Cristo. Toda la centralidad de la misión se fundó sobre unos conceptos de unidad de acción, de conocimiento mutuo y de universalidad, pero también de racionalidad, de adaptación y pragmatismo. La intención inicial fue la de enseñar a los nipones sus propios errores (Gómez Díez, 2007, p. 387).

A raíz de las desemejanzas tan evidentes percatadas durante los primeros contactos con los gentiles nipones, el designio de Valignano pasó a ser el de la superación de las diferencias, pese a que estas fuesen muy radicales. El italiano fue dispuesto a batallar contra la tiranía de la costumbre de los misioneros y del prejuicio (Lisón Tolosana, 2005, p. 160). Por otro lado, como observó Gómez Díez, "no había razón para esperar que ellos se acomodasen a las maneras de un puñado de extranjeros y, por tanto, se corría el riesgo de que al enjuiciar a estos como gente vil, conceptuasen de igual forma a su religión" (Gómez Díez, 2007, p. 371). Igualmente, el mismo padre Visitador, en uno de sus textos, había previamente comentado:

Porque o ellos no aprenden las costumbres y cortesías de Japón porque no quieren ni les parece bien, y esto es afrenta para ellos, y hacen contra razón pues ellos se han de acomodar a los japoneses, ya que vienen a su tierra y son pocos, y no los japoneses a ellos que no pueden dejar sus fueros, o no las aprenden porque no tienen ingenio y capacidad para eso, y entonces no conviene a los japoneses recibir ley y tomar por maestros hombres de tan poco ser (Valignano, 1944, nota 24).

Entre los japoneses y los misioneros, a menudo rechazados, existió toda una problemática debida a la manera de vivir occidental que permaneció activa en los jesuitas y que se presentaba como ajena a las costumbres del país. Los cristianos más honrados de Japón como Shimo, Don Protasio de Arima, Don Bartolomeo de Omura, pidieron constantemente que los jesuitas se asentaran al estilo de vida nipón. Aunque no fue fácil adoptar otras formas culturales, ya que se trató de algo mucho más peliagudo que 
un simple cambio de costumbres, el acercamiento tenía que empezar por un estudio más profundo del idioma y de la cultura local para que todo el proceso de evangelización fuera más eficaz. El mismo Francisco Javier insistió en la necesidad de estudiar la lengua japonesa para convertir a más gentiles. Valignano, antes de dejar Japón en 1582, se interesó más atentamente por el método de adaptación. Según él, el desprecio de los vicios de los japoneses -quienes para los padres no fueron respaldados por la Gracia de Dios- no era correcto ni apropiado. Es más, pese a que las costumbres fuesen muy distintas, creyó que tenían que ser asumidas por los padres jesuitas con tal de seguir adelante con la misión de evangelización (Gómez Díez, 2007, p. 371).

Como acto seguido, fue necesario revisar todas aquellas relaciones entre los misioneros y los colaboradores japoneses. Ambos tenían que estar al mismo nivel, sobre todo, en el trato con los superiores de la Compañía. Hubo que formar sacerdotes japoneses, es decir, un clero secular y también religiosos de la Compañía. Por tanto, resultó imprescindible la fundación de Colegios y Seminarios con cursos superiores e inferiores (Valignano, 1946, p. 25). El propósito principal de estos centros, cuyas enseñanzas abarcaron desde la religión a la teología, desde la mística al arte de occidente, fue el de armonizar las dos culturas, sin apartar los principios basilares de la Compañía de Jesús, pilares del mismo sistema educacional. Asimismo, propuso abrir seminarios para japoneses con el fin de enseñarles las virtudes y las letras y, al mismo tiempo, para que "después unos ayudasen a la Compañía entrando en ella, y otros se hiciesen clérigos o sirviesen otros ministerios" (Gómez Díez, 2007, p. 373). No cabe duda de que la introducción del arte y la cultura occidental fue el medio principal con que se intentó realizar desde el principio todo el catecumenado de la misión del Japón del siglo XVI (García Gutiérrez, 2012, pp. 1474-1478).

Al principio la vía de la adaptación resultó ser la más apropiada. Había que japonizarse para conseguir la cristianización y la evangelización de aquellas tierras. De manera que se dio lugar a la formación de algunas normas, bajo consejo de los japoneses, para el acondicionamiento a sus costumbres y a las formas de cortesías. Para empezar, Valignano recomendó a todos los superiores que enviasen misioneros que fueran expertos en el arte occidental, para enseñarlo a los nipones; en Teología, para solucionar cualquier tipo de duda que surgiera entre los neófitos, y que respetasen el 
aprendizaje y la observación de estas reglas. Con esa finalidad, como había previsto el padre Visitador, este "manual" se hubiera entendido solo en el mundo cultural "de llegada". Los superiores de la Curia romana, debido a su distancia, no fueron capaces de entender estas decisiones y, muy pronto, una vez que se percataron de lo que ocurría en aquellas tierras, decidieron contestar a lo que consideraron simplemente un ultraje a la pureza del Instituto. Sin embargo, el padre Visitador solo pidió a los padres jesuitas que entendieran y, a la vez, siguieran las manifestaciones culturales niponas para un correcto asentamiento a las costumbres y a la manera de vida japonesa (García Gutiérrez, 2012, p. 1476).

\section{Introducción a Lembranças para Japón}

En las páginas siguientes se propone el texto de las Lembranças para Japón, un manuscrito autógrafo e inédito del padre Gil de la Mata dirigido al padre General de la Compañía, y del que se conserva otra copia titulada Memoriale para Japón. Como explicó López Gay en su actividad literariocientífica, este manuscrito fue citado y poco estudiado por Alfred Schütte en Il Cerimoniale per i missionari del Giappone. Además, fue mencionado por Alvarez-Taladriz en una nota a las Adiciones de Valignano (Valignano, 1946, p. 126). No obstante, nadie se interesó en su transcripción, ni siquiera a la composición de un estudio más detallado acerca de estas sentencias. En efecto, este escrito reproduce la desaprobación de Roma a las decisiones adoptadas por la Compañía de Jesús en Japón y, en particular, a las de seguir adoptando el ceremonial y las formas de cortesías niponas.

Primeramente hay que aclarar una cuestión muy importante: no existe ningún manuscrito del Memoriale para Japón en portugués. Es oportuno especificarlo, ya que el padre Mata, pese a su conocimiento del idioma luso demostrado en sus últimas cartas autógrafas (López Gay, 1964, pp. 123131), al parecer redactó dos veces su escrito en castellano. Aunque se desconozca la motivación, todo hace pensar que se trata de otro caso de interferencia lingüística que a menudo aparece en los textos de los padres misioneros (Romo Alonso, 2011, p. 79). López Gay y otros estudiosos, en pasado, afirmaron la existencia de "una copia autógrafa de Mata, en portugués titulada Lèbráças par Japón”. Al contrario, como se demuestra más adelante, 
el manuscrito no se escribió en portugués, sino en castellano, mientras que se transcribió solo el título al idioma luso. Puede que el estudioso español, sin considerar el texto, se centró solo en el título del manuscrito del jesuita.

Sobre la vida del logroñés autor de las Lembranças, Gil de la Mata (también 'Egidio' por la traducción latina de su nombre; igual que su apellido se encuentra varias veces como 'Matta'), en varias ocasiones se trazó un perfil biográfico bastante completo en el que se especificaron varios detalles, desde su juventud a la misión de Japón (López Gay, 1964, pp. 79-122). Resumiendo brevemente su vida, se sabe que nació en Logroño probablemente en 1547 y que ingresó en la Compañía de Jesús el 12 de marzo de 1566. Llegó a ser sacerdote en 1576 y, en 1587, se instaló en Japón, haciendo su profesión solemne en noviembre de 1591. En 1594 viajó a Europa como Procurador de la misión de Japón y volvió a la tierra del Sol Naciente en 1598. Murió en 1599 por un naufragio ocurrido durante su segunda vuelta a Europa (López Gay, 1964, p. 79).

Su formación fue definitivamente la del jurista, dedicado al estudio de la Teología y de los problemas morales o de los "casos" de conciencia. Su carácter poco abierto no se prestó a la confrontación de ideas o al diálogo, aunque en todos los documentos acerca de su vida se subraya su prontitud y constancia en el trabajo. Gil de la Mata fue un conservador de las reglas de la Institución. Siempre mantuvo un aspecto particularmente servicial y totalmente entregado a su oficio; por otro lado, se dedicó a la formación de los japoneses que se habían convertidos al cristianismo como se comprueba también en la Historia de Japam de Luís Fróis:

Tomou o Padre o assumpto desta pia obra tanto de propozito, que foi notavel o frutto que por aquellas terras se fez: primeiramente cathequizando-os de novo, ensinando-lhes as orações, confessando-os, baptizando-lhes as crianças, instruindo-os nos rudimentos da fé catholica, tirando-lhes muitas ignorancias e abuzos que tinhão, ministrando-lhes o sacramento do matrimonio, tirando a muitos de peccado mortal [...] acudindo as suas necessidades espirituaes de noite e de dia com tanta solicitude e cuidado que os mesmos christãos se admiravão de ver a caridade e amor que com elles uza (Fróis, 1984, V, p. 144).

El logroñés se obsesionó con salvar lo legal y lo jurídico, las enseñanzas, las tradiciones, etc., de la Iglesia militante y del genuino espíritu de la Com- 
pañía de Jesús (López Gay, 1964, p. 120). Como afirmó López Gay, Gil de la Mata no fue un literato. Ni siquiera fue un autor de descripciones amenas, o fantasmagóricas, al igual que varios de sus compañeros. Su carácter, junto a sus ideologías y a su temperamento frecuentemente brusco, se opusieron con asiduidad al del padre Visitador Alessandro Valignano. El italiano a menudo dudó de las capacidades comunicativas del padre Mata, aunque apreció su diligencia y su constancia en el trabajo. El padre Visitador no llegó a entender al logroñés y su metodología de cristianización "ibérica" o "romana". En cambio, a partir de 1593, Valignano aprobó la fórmula de Matteo Ricci, alterada y adaptada por sus propias teorías que aplicó en el mismo Japón. Su programa de adaptación o, mejor dicho, de impulso a la acomodación y aceptación a los usos y costumbres japoneses se reveló mucho más novedoso y práctico. No temió arriesgar aspectos de las idiosincrasias occidentales a cambio de una mayor adaptación al universo japonés. Se trató de utilizar los conocimientos científicos, geográficos, etc., para atraer la atención y, al mismo tiempo, tener más crédito entre los gentiles (Gómez Díez, 2007, p. 367).

A tal propósito, hay que mencionar otro documento muy relacionado con el Memoriale para Japón, los Advertimentos e avisos acerca dos costumes e catangues de Jappão escritos por Alessandro Valignano en $1581^{1}$. Ambos textos circularon después del nuevo reglamento que entró en vigor durante la segunda estancia de Valignano en las tierras niponas. Los Advertimentos o Il Cerimoniale per i missionari del Giappone, (como fue traducido en 1946 al italiano) es un breve escrito práctico, fruto de la mirada intuitiva del padre Visitador, valorado positivamente por la Curia romana, y dirigido a todos los grados de la jerarquía de la misión en Japón. En la obra se establece cómo adaptarse conscientemente a la vida social del país; se tratan las formas de cortesía japonesas, el estudio diligente de la lengua, la adaptación a la comida, etc. Además, incluyó más de cien vocablos japo-

${ }^{1}$ Existe también una traducción al italiano: Alessandro Valignano, Il Cerimoniale per $i$ missionari del Giappone, "Advertimentos e avisos acerca dos costumes e catangues de Jappão" di Alexandro Valignano,... Importante documento circa i metodi di adattamento nella Missione giapponese del secolo XVI. Testo portoghese del manoscritto originale, versione letterale italiana... Edizione critica, introduzione e note di Giuseppe Fr. Schütte,... [Presentazione di Mgr. Celso Costantini.]. Roma, Edizioni di Storia e Letteratura, 1946. 
neses, y describió las reglas que los misioneros tenían que observar en el trato con los gentiles (García Gutiérrez, 2012, p. 1476). Este escrito nació como demostración y remedio a la ignorancia de las formas de cortesía japonesas que engendró, primero, una falta de prestigio y, en segundo lugar, un enfriamiento de las relaciones, sobre todo, por parte de los fieles.

La historia del Memoriale para Japón empieza con el periodo que Schütte denominó "Revisione in Giappone (1590-92)". Valignano ya había remitido el tratado de los Advertimentos a sus superiores. Posteriormente, consignó también una explicación sobre el primer capítulo de su escrito donde no negaba las dificultades y, a la vez, la mala interpretación de algunos padres, como Alonso Sánchez, quienes desviaron completamente el contenido del tratado. El padre Visitador prometió al General de la Compañía que durante su segunda vuelta a Japón hubiera revisado las instituciones y contemporáneamente el texto de las amonestaciones. El 2 de julio de 1590 comenzó su segunda visita en la tierra del Sol Naciente. No había transcurrido un mes cuando inauguró Katsusa, el 13 de agosto del mismo año, la Segunda Consulta general de la misión. Al parecer invitó a los superiores y a otros consejeros para discurrir sobre los asuntos de la Compañía; separadamente, convocó a algunos hermanos japoneses a los que habló en su idioma.

La alocución con que dio inicio a la reunión exponía el propósito del convenio: se trataba solo de una consulta, ya que la Congregación provincial se hubiera reunido a la vuelta del viaje de Valignano después de su visita a Hideyoshi. El "tirano", como le apodó Gil de la Mata, con su decreto anticristiano del 25 de julio de 1587 confundió la situación entre los jesuitas por su carácter incierto. No obstante, según declaró el padre logroñés en su Relación del estado de Japón ${ }^{2}$, los oficios de los jesuitas siguieron adelante y sin particulares problemas. Valignano intentó sedar la persecución de los cristianos con su visita al Kambaku y con la entrega de regalos preciosos enviados por parte del Virrey para homenajearle. Durante la larga espera, esperando la respuesta de Hideyoshi, el padre Visitador instauró una comisión con otros miembros de la Compañía, como el secretario de la Consulta, Luis Frois, Damian Marín y otros dos diputados, Pedro Gomes y Organtino

${ }^{2}$ Archivo Romano de la Compañía de Jesús (ARSJ), Congregazioni (46, 485-488). 
Gnecchi-Soldo. Además, Francisco Pasio quien fue elegido «socio» del Visitador.

La temática central de la Consulta, que se avivó durante la tercera reunión, concertó la posibilidad de salvaguardar y observar totalmente las reglas del Instituto y los principios de la Compañía en aquella situación de Japón y, por tanto, diferente de Europa. Finalmente, hubo una unanimidad a favor de una revisión de las primeras disposiciones de Valignano, tanto por las experiencias realizadas, como por la situación de la Iglesia sujeta a las persecuciones. El 5 de noviembre de 1590, Valignano constató que las Regulae generales y las Regulae communes ya habían sido revisadas. Quedaba claro, por tanto, que estas normas se tenían que observar incluso en Japón. Por otro lado, las Reglas permitían cierta flexibilidad, sobre todo en aquellos casos de necesidad. Mucho más complicada parecía la observación de las Reglas de oficio en las que los padres tenían que conformarse a la manera de vivir en Japón. Cada regla debía ser revisada y elaborada; entre estas, también los Avisos dos costumes e catangues de Japão. Antes de ser transmitido al padre General, el mismo texto necesitaba una ulterior modificación.

Otro punto ampliamente debatido en diferentes encuentros fue la adaptación a las formas de cortesías japonesas. Al principio, la acomodación pareció un asunto que no se podía discutir ni alterar. A partir de la quinta reunión, conjuntamente a la llegada de una carta de Claudio Aquaviva, General de la Compañía, en la que trató sobre esta misma cuestión, la adaptación empezó a ser una problemática que necesitó una modificación seria y a la que había que aportar varias limitaciones. Como afirmó el investigador Arimichi, los jesuitas "debían de realizar sus funciones litúrgicas con reverencias y decoro, exactamente a como hacían los bonzos" (Arimichi, 1971, p. 128). En la minuciosidad de los Avisos, Alessandro Valignano recomienda a los misioneros incluso el uso a lo bonzo de los movimientos del cuerpo, para mostrar respeto, distancia, cercanía, etc. Describe también los zaxisquis -estancia al modo japonés- para recibir y agasajar a los huéspedes según su calidad; o, incluso, los regalos y presentes que, según el italiano, lubricaban las relaciones sociales (Lisón Tolosana, 2005, pp. 156-160). Sin embargo, el mismo Aquaviva, en una misiva de 1585, parece desconfiar de la imitación de los bonzos. Según el padre General la emulación fue sinónimo de falta de pobreza y virtud, y ponían en peligro la identidad de la 
propia Compañía (Gómez Díez, 2007, pp. 374-375). De hecho, en varias ocasiones, ocurrió que la acomodación de Valignano resultó a muchos padres exagerada y contra los principios de la Institución. La asimilación de las formas de cortesías japonesas fue aclarada con un preciso reglamento; en primer lugar, se empezó a definir el número y el género de los colaboradores en las casas y durante los viajes de la misión. En segundo lugar, se suprimieron objetos y hábitos típicos orientales que habían sido aceptados por los jesuitas durante la misión.

Alessandro Valignano concibió la asimilación al ceremonial japonés como una "prudencia religiosa", una sutil manera de vivir y no un método para adquirir mayor autoridad. En realidad, su intención fue la de filtrar el Evangelio, volverlo más fácil, "depurarlo de excrecencias europeas y transportarlas en esencia a la cultura japonesa” (Gómez Díez, 2007, p. 374). Además, el cumplimiento de estas reglas dependió estrictamente de los colaboradores, cuyo número fue limitado según las necesidades que surgían, con lo cual no todos pudieron cumplir determinadas acciones u oficios. La observación de esta jerarquía social fue el punto más importante de la asimilación decretada por Valignano. A partir de este momento aumentó la necesidad de colaboradores que posteriormente se criticó en el mismo Memoriale. Sin embargo, Valignano reguló la distribución de estos asistentes. En primer lugar los grandes centros, es decir, los Colegios o los Seminarios (donde el número de personas era aproximadamente de diez) podían disponer de tres o cuatro dōjuku, de dos rapados y de un tonobara. Estos colaboradores tenían también que recibir y atender a los visitantes, comunicar mensajes y desarrollar varias funciones en la Compañía. Uno de los rapados se encargaba de la ceremonia del chanoyu; en cambio, el tonobara preparaba el sakana ${ }^{3}$ y otras comidas. Otro tipo de trabajo como los de la cocina, la colada, el transporte de cargos, etc., requerían de unos sirvientes o, como los denominó Mata, "mozos". El mozo también custodiaba los zapatos o las sandalias al padre misionero que las dejaba en el umbral de la puerta de casa, como es todavía costumbre en Japón quitarlas antes de entrar (Valignano, 1946, p. 46).

${ }^{3}$ Un entremés preparado con sake. 
La concesión de los colaboradores fue un asunto reservado al padre Viceprovincial. En las residencias menos importantes con un par de dōjuku y un rapado tenía que ser suficiente. Para las misiones, sobre todo en los viajes presenciados por los Superiores, el padre Viceprovincial representaba la autoridad eclesiástica de Japón. Sus funciones fueron de varios géneros. Se encargaba de comunicar y tratar con diferentes tipos de hombres, en particular con la nobleza, los príncipes, etc., quienes debían de promover la conversión en su propio territorio. El Viceprovincial podía ser acompañado, durante sus visitas a las iglesias, por un hermano japonés, quien se encargaba de predicar y hacer catequesis. Otro laico japonés desarrollaba el papel de secretario, preocupándose de escribir correctamente. Y, por último, un hermano laico europeo que cuidaba de la administración. En los casos en los que hacía falta, se podía incorporar al grupo de los colaboradores también un intérprete. Según Valignano, ese conspicuo número de personas, el cual para Europa constituía casi una exageración, era extremadamente necesario para la realización del perfecto ceremonial japonés que, de ninguna manera, podía descuidarse, en particular para los príncipes feudales. Se necesitaba, por tanto, al menos un dōjuku para preparar el altar, un rapado para la ceremonia del chanoyu y para los mensajes, dos tonabaras para recibir a los visitantes, servir a la mesa, trabajar en la cocina, etc., y también algunos «mozos» necesarios para los trabajos más humildes. El Viceprovincial no podía actuar sin la presencia de otras personas. Igualmente, se necesitaban una o dos personas más para los viajes a las regiones más remotas. Además, quedaba claro que si un padre Visitador viajaba a Japón, el Viceprovincial tenía que someterse a su criterio.

En las Regulae generales, además, se estableció que en los territorios de Bungo, Miyako y Shimo los superiores requerían un irmão japonés para las prédicas y las catequesis; de un dōjuku para el altar, las comisiones, el sakazuki ; y de dos servidores para la cocina, el caballo, etc. Asimismo, cuando los irmãos viajaban, solo tenían que ser acompañados por uno o dos servidores. El relevante número de los colaboradores de ninguna manera expresaba vanidad o pompa, sino solo el ceremonial para recibir a los visitantes. Además, era directamente proporcional según el rango social de cada uno de ellos.

\footnotetext{
${ }^{4}$ Ceremonia del sake. Concretamente, es la copa con el que se bebe el sake.
} 
Por otra parte, siguiendo el texto manuscrito, se ordenó que el número de los colaboradores según las autoridades romanas tenía que ser categóricamente reducido. Toda esta información que llegaba desde Japón, de alguna manera tuvo un impacto negativo en la Compañía y, en particular, en la opinión del padre General Claudio Aquaviva. La noticia de que los misioneros fueron obligados a tener un dōjuku o un propio servidor no debió de gustar al italiano. La otra cuestión que surgió en este periodo fue la asignación fija de los dōjuku a cada misionero. Para mantener un cierto respeto hacia estos colaboradores, se prohibieron los continuos cambios de los dōjuku a los jesuitas: ellos desearon la custodia de un mismo padre que le conociera y le guiara para poder entrar definitivamente en la Orden. No resultaba digno cambiar de persona en persona, ni siquiera permitía adquirir una educación necesaria para su formación moral. Tampoco los mismos cristianos veían positivamente estas sustituciones. Para Valignano, lo esencial era que el dōjuku obedeciera al padre superior local, la única persona con el poder de asignarle los cargos, castigarle o cambiarle a otro padre si lo consideraba necesario, aunque tenía que demostrar a las autoridades la razón de esta sustitución.

\subsection{El texto manuscrito}

Las órdenes de Claudio Aquaviva, escritas de puño y letra por el padre Gil de la Mata y tituladas Lembrámças para Japón, se enfrentaron al proceso de inculturación perseguido por Valignano. En realidad, se pueden considerar como una franca defensa a favor de la preservación del Instituto que tuvo que primarse y, a la vez, anteceder la adaptación de las "catangues" japonesas. El manuscrito que se preludia releva casi un tiempo pasado de la Compañía, y roza el gobierno del P. Francisco Cabral, segundo superior de la misión, el cual duró desde 1570 hasta la mitad de 1581. Durante esta época se eliminaron varias costumbres japonesas que ya se habían incorporado a las idiosincrasias de los misioneros en Japón. Entre ellas, por ejemplo, se abolieron la ceremonia del té, los vestidos al estilo de los monjes, etc. Cabral "creyó que los japoneses debían de ser conducidos por el camino férreo de una subjección tal que lograran extirpar sus vicios y pecados" (López Gay, 1986, p. 67). De este modo, algunas concesiones de Valignano 
sobre determinados asuntos domésticos -que ya se indicaron en la sección anterior- y la cuestión de la pobreza, fueron notablemente restringidas. Es cierto que el verdadero iniciador de la adaptación a las costumbres japonesas fue el padre Cosme de Torres (1510, Valencia - 2 de octubre de 1570, Amakusa), sucesor de Cabral quien, como es notorio, paralizó el proceso de evangelización por su desconfianza hacia los nipones. La verdad es que, tras haber constatado las dificultades lingüísticas y lo difícil que resultó a los misioneros "comer, vestir e dormir como los japoneses", el padre Torres desde el principio consideró necesaria la familiarización con el universo japonés (Gómez Díez, 2007, p. 369).

En todo caso, como escribió Schütte, en Japón se temió que las Constituciones de la Orden pudieran ser dañadas u ofuscadas por la adaptación a las costumbres japonesas. Con este tipo de escrito se fueron remarcando los principios originarios de la Compañía, ya que las numerosas relaciones sobre las tierras orientales habían sido muy generosas y más que positivas en las descripciones. La experiencia personal de cada jesuita y las relaciones escritas no podían relatar una verdad absoluta, capaz de ilustrar una situación detallada de cada cosa.

Parece ser que el padre Mata no llegó a entregar el Memoriale al padre Visitador. Como confirma una carta del 12 de diciembre de 1596 de Valignano al P. João Alvarez, durante su viaje desde Italia a España, precisamente en el golfo de Lion, al padre logroñés le sorprendió una tormenta por la que perdió todos los documentos (Valignano, 1946, p. 76). Después de informar al General de lo acontecido, y no pudiendo volver a la Curia romana, al parecer refirió todo lo que había redactado a Valignano, anunciándole las líneas generales y una hipotética aproximación al contenido de este manuscrito. Por otro lado, el padre Visitador, consideradas las amonestaciones y los acosos de alejamientos a las reglas del Instituto, contestó al mismo Aquaviva, tranquilizándole sobre el valor de sus hermanos, según él, auténtico "oro fino" de la misión en Japón. La entereza de los jesuitas en Oriente era evidente, como anunció Valignano, ya a partir de las numerosas persecuciones a las que fueron sujetos los padres, al igual que la mucha abnegación, la fatiga y las privaciones que tuvieron los misioneros de la Compañía. La carta que Valignano envió el 26 de noviembre de 1596 desde Goa al General de la Compañía, realmente fue una respuesta al informe oral que le hizo Gil de la Mata. Todos los puntos evidenciados en el 
manuscrito se trataron en una Consulta general, pese a que el padre Visitador no contestó inmediatamente. Valignano decidió esperar la elección del Procurador que tenía que ser efectuada durante la segunda Congregación provincial, y en la que fue elegido por segunda vez el padre Gil de la Mata. Como es notorio, en febrero de 1599, durante su segunda vuelta a Roma, además de los documentos el jesuita español perdió también la vida. Por consiguiente, aquellos informes y las actas de la segunda Congregación provincial, es decir, la respuesta (al Memoriale) que Valignano mandó a Roma, se perdieron (Valignano, 1946, pp. 76-77).

En el fondo las Lembrámças se casan perfectamente con las ideas conservadoras del padre Mata, quien debió de influenciar toda la redacción del texto ideado por Aquaviva. En el encabezamiento del manuscrito se lee que las Lembranças para Japón son la "respuesta al artículo 5 de la Congregación de Japón”. Pues bien, antes de empezar con el análisis del texto original, es oportuno transcribir el $5^{\circ}$ artículo de las Constituciones, extraído de la redacción de 1550, para comprender algunos detalles del texto manuscrito de Gil de la Mata que se propone más adelante:

V.- Todos los compañeros, puesto que han de ser presbíteros, estarán obligados a rezar el oficio divino, según el rito común de la Iglesia, pero en particular, no en común o en coro. Y en todo lo que se refiere al comer, vestir y las demás cosas exteriores, seguirán el uso común y aprobado de los buenos sacerdotes. Así, lo que cada uno quitare de esto, por su necesidad personal, o por deseo de provecho espiritual, lo ofrecerá a Dios por devoción y no por obligación, como ofrenda razonable del propio cuerpo. Esto es lo que, sometido al beneplácito del ya dicho señor nuestro Paulo, y de la Sede Apostólica, hemos podido explicar, a modo de imagen de nuestra profesión. Lo hemos hecho ahora, para informar sumariamente tanto a los que nos preguntan sobre nuestro género de vida, como también a nuestros sucesores, si Dios quiere que tengamos en alguna ocasión quienes nos sigan por este camino. Y como hemos experimentado que éste lleva consigo muchas y grandes dificultades, nos ha parecido oportuno establecer que nadie sea admitido para hacer la profesión en esta Compañía, sin que su vida y formación intelectual hayan sido probadas con largas y diligentísimas probaciones, como se declarará en las Constituciones. Porque, en realidad, este Instituto exige hombres del todo humildes y prudentes en Cristo, y señalados en pureza de vida cristiana y en letras. Más aún, también los que se admitan para 
coadjutores, en las cosas espirituales y en las temporales, y para escolares, no serán admitidos en esta milicia de Jesucristo, sino cuando hayan sido examinados diligentemente, y hallamos idóneos para el mismo fin. Unos y otros, hechas las probaciones suficientes, y cumplido el tiempo establecido en las Constituciones, para devoción y mayor mérito, harán sus votos, pero no solemnes, sino tales que los obliguen mientras el Prepósito General juzgue que ellos continúe en la Compañía (según se explicará más ampliamente en las Constituciones), excepto algunos que, por su devoción y por la calidad de la persona, puedan hacer, con licencia del Prepósito General, los tres votos solemnes. Dígnese Jesucristo favorecer estos nuestros débiles comienzos, a gloria de Dios Padre, al cual se dé siempre toda alabanza y honor por los siglos. Amén (Arzubialde et al., 1993, pp. 39-40).

A raíz de este artículo, es muy probable que la base para el escrito de Aquaviva redactado por Mata se construyera sobre dos nociones: la primera, "se refiere al comer, vestir, y las demás cosas exteriores, seguirán el uso común y aprobado de los buenos sacerdotes". Y la segunda, que "nadie sea admitido para hacer la profesión en esta Compañía, sin que su vida y formación intelectual hayan sido probadas con largas y diligentísimas probaciones”. El manuscrito hace hincapié en estos dos puntos y, por consiguiente, en el texto de la Constitución para reforzar lo que se expresa. En realidad, quien escribe busca en el estatuto la auctoritas para dar mayor corpulencia a su discurso. Por otro lado, si se considera el mismo término 'memorial', según el significado que se le daba, se nota que en su esencia la palabra no es más que una "petición que se da al juez, o al señor para recuerdo de algún negocio”, como anotó Covarrubias (1611, p. 1129).

El Memoriale expone unas órdenes esparcidas y, por tanto, sin una precisa estructura temática, dirigidas al padre Visitador Alessandro Valignano. Lo cierto es que algunos preceptos parecen ser esbozos dictados por el mismo Aquaviva y recopilados por la pluma de Gil de la Mata. En cambio, otras advertencias vuelven a considerarse a lo largo del texto con más detalles y poca diplomacia. El lenguaje es claro, neto y sin rodeos, dirigido hacia un cambio radical y situacional. La mayoría de las resoluciones afectan al comportamiento y al oficio del Visitador. El primer dictamen que repentinamente se subraya en las Lembranças apunta al número de los colaboradores del padre Visitador, aspecto ampliamente reducido: el mismo 
superior podía tener solo tres colaboradores -entre ellos solo un dōjuku y un hermano japonés que conocía tanto el idioma nipón como el portuguésy de los mejores que había en Japón. Valignano podía contar también con dos servidores y cuatro caballos. Anteriormente, el padre Visitador había regulado las salidas con caballo solo para moverse en los largos recorridos. Además, los padres no se tenían que servir del nuricaqui (norikake), que era un caballo para carga muy poco conveniente y, a los ojos de los gentiles, bastante ridículo por ser un medio que ellos mismos empleaban. Por otro lado, quedaban totalmente excluidos los tonobaras ${ }^{5}$ por desarrollar un papel muy parecido al de los bonzos. En las últimas órdenes del primer precepto se prohibió también al compañero del padre Visitador tener otros servidores; igualmente, la elección de los dōjukus tenía que ser establecida, al igual que su escisión, por el superior. Quedó vetado también el aprovechamiento de los niños menores de 18 años para cualquier tipo de oficio. De este modo, según cuanto se explica, se evitaban inconvenientes con los servidores mayores. Estos últimos tenían que obedecer solo al padre Visitador y a su compañero, mientras que no dependían del hermano japonés ni del dōjuku. Nadie de la casa podía servirse de ellos para los trabajos de las residencias con excepción de los superiores.

El dōjuku fue otra figura objetivo de las restricciones de Aquaviva. Este personaje exhibía un aspecto muy parecido al del religioso; además podía ser intérprete, preparar el altar, ayudar en los entierros, etc. (Valignano, 1946: 46). En el periodo anterior a la llegada de Valignano, este personaje constituyó uno de los elementos más innovadores. Tras observar que los dōjuku asistían a los bonzos durante las enseñanzas, además de dormir con ellos; los misioneros decidieron hacer lo mismo, de manera que, alrede-

${ }^{5}$ Según Esquivel el tono o tonobara era un "hombre escudero menos que hidalgo, y caballero" (Esquivel, 1630). Asimismo, Valignano definió a los tonos como: "los que mandan y señorean la tierra; entre los cuales hay mucha diferencia de dignidades y preeminencia, como son entre nosotros los condes, marqueses y duques" (1954: 8-9). De hecho, además de los rapados (los dōjukus), los misioneros podían contar con el apoyo de estos "laicos de noble familia". Sin embargo, como atestigua el manuscrito, el padre general Aquaviva llegó a prohibir la colaboración con los tonos dentro del Instituto. 
dor del 1600 los jesuitas contaban con 200 dōjuku. Además, en la primera Congregación Provincial, los dōjuku llegaron a ser admitidos en la misma Compañía de Jesús (Gómez Díez, 2007, p. 373).

Según las disposiciones redactadas por Mata, el superior debía mostrar cierta preponderancia y nunca una sumisión a su colaborador japonés, aunque jamás se les podía golpear para reprenderle, a no ser que fuera un hermano mayor de edad o un bonzo anciano. Incluso, nunca podían dormir dos o tres dōjuku juntos y a solas, sino siempre tenían que ser acompañados por otro hermano. En esta última decisión se intuye quizá un cierto temor a que los japoneses pudieran conspirar contra los jesuitas o causar problemas similares. De todas formas, no podían disponer de los servicios de los dōjuku las personas que no practicaban el cristianismo en tierra nipona, como los Rectores de los colegios o los lectores; mientras que un hermano siempre debía tener cuidado con ellos.

A partir de la sexta advertencia, en el Memoriale se analizan con mayor detenimiento los objetos que pertenecían al ceremonial de los japoneses. Se introducen unas limitaciones que interesan algunos objetos de la vida cotidiana en el Japón de la época. En consecuencia, se ordena al padre Visitador un estilo de vida más occidental, sin el empleo de objetos pertenecientes a la cultura de llegada, como por ejemplo el chanoyu que tenía que sustituirse con el común té de casa, sin la ceremonia del bonzo. De igual manera, los padres no tenían que utilizar bento para llevar platos y comida, sino simples cajones que tenían en las residencias. Al mismo tiempo, no tenían que llevar abanicos y otros objetos cuyo empleo podía molestar a los europeos si se enteraban a través de las relaciones. Se prohibieron incluso las cartas escritas y los regalos al padre Visitador o algunos de sus compañeros. En el manuscrito también hay toda una serie de instrucciones para los viajes y los objetos que no se tenían que llevar en las visitas, entre ellos, relojes despertadores y ornamentos para las misas. Los padres tenían que parecer bastante sobrios, sin aparentar riqueza ni ostentación. A la vez, tenían que ser neutrales y no mostrar una aceptación total a las costumbres japonesas como, por ejemplo, la del té molido con la escudilla.

Otra temática ampliamente debatida en muchos documentos es la de la vestimenta de los jesuitas en Japón. Se cuentan varios testimonios que tratan sobre esta adaptación externa por parte de los jesuitas. El mismo 
Valignano en el Sumario de las Cosas de Japón (Valignano, 1954, p. 231) afirmó que "al principio, los nuestros, para tener con los japoneses alguna entrada, se vistieron de seda”. Al parecer, Aquaviva no aceptó esta costumbre y, por tanto, lo prohibió incluso en la ciudad de Miyako. Solo podían llevar seda los seculares. Nadie de la Compañía podía vestirse a su propio gusto sin respetar las reglas de Europa, con que todos tenían que llevar los manteos, o, el dôbuku cuando se iban de viaje (Valignano, 1946, p. 45). Por otro lado se prohibió a los hermanos japoneses llevar los bonetes típicos de los jesuitas.

Entre el padre Visitador y los compañeros debía existir cierta distancia, primero, no aceptando favoritismos en ninguna de las dos partes; en segundo lugar, manteniendo una igualdad entre los componentes de la Institución en Japón. La palabra de Dios tenía que ser su unión, su símbolo de reconocimiento a la hora de las visitas de los japoneses. Lo que realmente importaba era que los nipones se dieran cuenta de que la presencia de los jesuitas en la tierra del Sol Naciente solo se debía a su intención de "ganar almas" convirtiéndolas al cristianismo. El rigor de la doctrina no solo se debía demostrar externamente sino que los propios jesuitas tenían que respetar unas series de reglas firmes. Los superiores y los padres no tenían que criticar ningún tipo de asunto. Se primaba el esfuerzo de los misioneros que confesaban y que ayudaban a las almas a través de aquellas reglas básicas de la Compañía. El mismo catecismo fue el mejor reflejo del esfuerzo de adaptación por parte de los jesuitas que intentaron convertir a los gentiles a la fe de Cristo (Gómez Díez, 2007, p. 372).

El Memoriale termina con una evidente crítica hacia la aceptación de las "catangues" japonesas promulgada por Valignano en su Cerimoniale, al parecer, una reflexión sobre las experiencias funcionales que quizá resultó a los padres demasiado optimista por la manera de apuntar al futuro de la religión en Japón. Tanto los superiores como los inferiores tenían que preservar aquellas reglas del Instituto y no adaptarse ni hacer lo que a los japoneses más les contentaba, en particular, si contradecía los principios de la Orden. Por otro lado, lo que más asustó al cardenal Aquaviva, que lo había aprendido en las relaciones que llegaban desde Japón, era la facilidad con que se podían introducir las cosas de Occidente. El General de la Compañía temía que los jesuitas pudieran tratar cosas del Estado japonés 
y, por tanto, prohibió la trata de esclavos o la intercesión de los frailes en asuntos familiares. Había que introducir la santa ley paulatinamente, y eliminar paso a paso las dificultades que los misioneros encontraban en el camino de la ganancia de las almas.

\section{Forma externa y transcripción}

El clásico papel blanco japonés del manuscrito presupone que el texto fue redactado en el mismo Japón y no después de 1595, al igual que la Relación de los Reinos de Japón incluida en la misma sección del Archivo de los jesuitas en Roma, las Congregazioni n. 46. Las Lembranças para Japón se colocan entre el f. 401 y f. 402 y se guardan en perfecto estado. Tan solo se hallan algunas partes perforadas por la humedad o debido a las tachaduras del mismo autor. Pese a que las escrituras de las dos caras de cada folio se solapen, la lectura y el desciframiento del código antiguo es bastante clara. Las normas expuestas en las Lembranças están divididas en pequeños párrafos que se han intentado respetar y reproducir en la transcripción exactamente según aparecen en el texto. La única modificación aportada al texto ha sido la modernización de algunos términos para que la lectura sea más agradable y amena. Por consiguiente, se han eliminado las complejas abreviaturas (excluida 'V.P.') y se ha cambiado el uso de las letras $i, j, u, v$ según la ortografía moderna. Por otro lado se ha respetado, por lo general, la puntuación -aunque cuando el texto lo ha necesitado se siguió el uso moderno- y se han cambiado algunos términos arcaicos empleados en el siglo XVI. Por último, la única abreviatura relativa al apartado crítico adoptada es: “[?]”, en caso de duda con la descodificación del término.

Finalmente, el documento que se transcribe a continuación, además de su valor histórico y cultural, es otro testimonio del continuo intercambio léxico que se produjo entre las lenguas occidentales, en este caso el castellano y el japonés (Romo Alonso, 2011, p. 79). En particular, llaman la atención los términos japoneses mencionados por Mata (dôbuku, chanoyu, etc.) y que fueron recibidos como neologismos en español a través de textos como el que se presenta. 


\section{Ihs \\ Lembranças para Japón (Respuesta al art. 5 de la Cong. Japón)}

- [f.401] Para el padre V. P6 . bastan tres compañeros. Un padre, un hermano, y un dogico ${ }^{7}$ que sirva a ambos, y estos escogidos los mejores de Japón, de manera que el hermano sepa la lengua y pueda escribir japonés ${ }^{8}$ y portugués, y ayudar al padre compañero en su oficio. Dos mozos de servicio que andan a pie para servir a los cuatro. Cuatro caballos con sus cuatro mozos, y esto para cuando van fuera. Cuanto a los tonabaras omnino se excusan, así para el superior como para los collegios, porque los bonzos hacen este oficio con igual autoridad, y cuando no los hay en casa, no faltan de fuera para recaudos, o para hospedar persona principal 9 .

\footnotetext{
${ }^{6}$ Abreviatura de 'Vestra Paternitas' empleada en la correspondencia y en los escritos por los jesuitas para indicar al padre General y solo él.

${ }^{7}$ Cfr. Carles E. O’Neill, S. I., Joaquín M ${ }^{\text {a }}$ Domínguez, S.I., Diccionario histórico de la Compañía de Jesús, Biográfico-Temático, Vol. II, Madrid, 2001, 1134-1135: "El Vocabulario de la língoa de Iapam (Nagasaki 1603 f 73), compuesto por varios jesuitas del Japón, da esta definición: "Dōjucu. Moços ou gente rapada que serve aos Bonzos nas teras [templos budistas]". Los dos ideogramas de la palabra japonesa admiten otra versión latinizada en el mismo Vocabulario f. 74: "Dōxucu. Vonaji yado. A mesma pousada, ou pousar na mesma casa". Dōjuku y catequista se distinguían en que este era un oficio realizado a veces por mujeres, mientras que döjuku era un estado de vida (JapSin 55 69), aunque no necesariamente perpetuo, al que se admitía solo a varones. La palabra dōjuku más que un término budista era un nombre común de la lengua japonesa adoptado por los bonzos para denominar a los niños de sus escuelas o teragoya. A los niños y jóvenes que vivieron desde 1550 con ellos en régimen de internado los llamaban "moços de casa", "rapados", "mininos japōes daqui de casa", etc. no dōjuku (dojuco, doyuqu, doxico, dogico, etc., con pronunciación portuguesa) fue adoptada hacia 1579, antes de que Alessandro Valignano fundara los seminarios de Arima y Azuchi. Con ella se apelaba a los niños, jóvenes y adultos célibes, algunos con voto de castidad, comprometidos con la CJ para el apostolado. Se le reconocía por su cabello rapado y su traje talar distinto de la sotana de los jesuitas. Ya durante el superiorato (1550-1570) de Cosme de Torres estudiaban su lengua nativa e instrumental, etc., con miras a la apologética, la predicación y el trato social. Desde 1580, los dōjuku niños y adolescentes, incluidos algunos chinos, portugueses, coreanos y mestizos, siguieron recibiendo en los seminarios una esmerada formación religiosa y humanística. Se le dio nuevo impulso a la música, pintura, grabado y otras artes, que entraron de lleno en su vida diaria".

${ }^{8}$ En el manuscrito pone "Japón", en este caso se prefiere facilitar la lectura.

${ }^{9}$ Siguiendo el Ceremonial, esta misma disposición parece ser precedentemente adoptada durante los viajes de los misioneros: "E conveniente che, se i Padri escono a viaggiano da una località all'altra, abbiano con loro per quanto è possibile, un Fratello, un Dōjuku e due servi. I Fratelli siano accompagnati da un servo nella città in cui abitano, in altri luoghi da due servi” (Valignano, 1946, p. 48).
} 
- El compañero del padre V. P. no tiene necesidad de otros mozos [borra una silaba] para caminho ${ }^{10}$, en casa si ocurre necesidad se sirva de los que hay.

- Ninguno [borra una palabra] ni aún de los que están en residencia ha de escoger su propio dogico, sino el superior inmediato lo diera y quitara a cada uno.

- De cómo nos [probable falta por 'los'] pequeños menores de 18 años no se use en ninguna parte (son estos unos niños que traen caballo, $\mathrm{y}$ toman los zapatos, y sirven de aguamanos, y de servir a la mesa y se pueden hallar inconvenientes con los mozos grandes.

- Los hermanos no tengan mozos, ni usen de los de casa ${ }^{11}$, sin licencia del superior.

- No sirvan los dogicos para barrer las cámaras, y hacer o concertar camas, sino cada uno concierte la suya, conforme a la regla.

- No se use lavar los dogico, o mozo alguno los pies a los nuestros, cuando fuere necesario un hermano lo puede hacer, a quien el superior lo encomendare.

- Cuando en las residencias hubiere más de un dogico, duerma el hermano con ellos en una pieza, y cuando no hubiere más que uno duerman todos los 3 en una sola.

- [f.402a] Que ni el superior universal ni otro de los nuestros anden en silla o en cosa en que vaya a hombros de otros ${ }^{12}$.

- Ningún superior universal, ni particular tenga en las mesas más distinción, que del primer lugar, no usando de mesas más altas que los otros.

- El V. P. de Japón no tenga chanoyu ${ }^{13}$ (que es la casilla donde está el cha con todas sus vasos y aparejos) ni bonzo para este efecto, más sírvase del común de casa, y cuando van camino no es necesario llevar este oficial.

- Los padres no tienen necesidad de traer bento ${ }^{14}$ (que es un cajón de madera en que están ${ }^{15}$ los platos $\mathrm{y}^{16}$ y la comida) es cosa nueva y de

${ }^{10}$ Se deja el término en portugués como se encuentra en el manuscrito en lugar de traducirlo al castellano 'camino'.

${ }^{11} \mathrm{El}$ autor borra la construcción "como de propios".

${ }_{12}$ Pese a que Valignano limitó el empleo de estas sillas, coxi o koshi (1946, p. 49), ahora quedaba totalmente prohibido.

${ }^{13}$ Ceremonia japonesa del té.

${ }^{14}$ Ración de comida sencilla preparada para llevar, bastante común en la cocina japonesa.

${ }^{15} \mathrm{El}$ autor borra la palabra.

${ }^{16}$ Repite la conjunción. 
que nunca usaron ${ }^{17}$, más sírvanse de los comunes de casa, y cuando fueren camino largo el superior los acomode.

- Cuando se mudan los padres de una casa a otra, no lleven consigo relojes, despertadores, ni ornamentos para decir misa, y cuando fueren necesarios ornamentos para algún viaje los procuradores prueban [?], ni muden otras cosas que se hallan en el lugar para donde ven. Y así boyones y otros instrumentos de cha son desnecesarios, porque están prohibidas las residencias, cuando mucho el cha molido con la escudilla.

- No pueda traer seda ningún hermano aunque sea en el Meaco, y aunque sea para llevar recaudo a algún nuestro, más llévalo persona secular bien vestida.

- Que ningún superior permita, ni de facultad a ninguno de la Compañía particularmente a hermano ${ }^{18}$ japonés que haga el vestido a su gusto, ni consienta que en nada discrepe del modo de la Compañía, (sacando el tiempo de persecución) y que los hermanos anden dentro y fuera con bonetes ${ }^{19}$ nuestros, y que vayan siempre acompañados con personas de confianza. Y generalmente se procuren introducir, a lo menos en los padres nuestros, manteos por vestido, ordinario, como en Europa.

- Ningún padre que no tenga oficio ordinario de cultivar cristiandad tenga dogico para su uso, ni para que le ayude ordinariamente a misa, o a otras cosas, como $^{20}$ son los Rectores de los colegios, y seminarios, y lectores, ni tampoco como nos, sino en todo se hayan como en Europa, si alguna vez fueren fuera pidan al superior compañero, o si fueren superiores ellos los tomen de los que sirven a la comunidad, aunque este sea en algún detrimento de los dogicos, lo cual se podrá suplir habiendo siempre un hermano que tenga cuidado de ellos, y los ocupa.

- Que ni los padres ni hermanos usen de abanos y ventallos, pues en la India con ser tierra más cahíta [f.402b] no se usan, ni resultara detrimento a la cristiandad. Y sabido en Europa ese uso se desedificarán y murmurarán.

- Que ninguno escribiera al padre o hermano compañeros del padre V. P.. o a sus consultores. Que le negocien alguna cosa con el superior, sino que lo propongan con [...] al mismo superior inmediatamente.

${ }^{17} \mathrm{El}$ autor borra la palabra.

${ }^{18}$ En el manuscrito pone "Japón".

${ }^{19}$ Según el D.R.A.E: "Especie de gorra, comúnmente de cuatro picos, usada por los eclesiásticos y seminaristas, y antiguamente por los colegiales y graduados".

${ }^{20} \mathrm{El}$ autor borra la palabra. 
- Que ninguno de la Compañía pueda dar el padre V. P. de Japón, o a sus compañeros o a sus consultores algún presente, fuera de las cosas menudas que todos pueden dar o recibir.

- Que de tal manera se haya el V. P. de Japón con sus compañeros, y con todos los demás en conceder, o negar, y en acomodarlos, o tratarlos, que ni los de casa ni fuera puedan hacer concepto ni decir que uno prima con él más que otro, ni que es más rico que otro.

- Que se procure en Japón a todos los que vienen a visitar los padres hablarles de Dios, y que vayan haciendo concepto que los padre no gustan de sus visitas por obligarlos a eso, sino por ganar sus almas, y que se vayan instruyendo que han de dar antes la vida que tornar atrás ef. [?] y que en esa materia no es cosa leve, sino muy grave el mentir.

- Que no se meta entre los nuestros lenguaza de notar faltas de otros ni por vía de gracia, particularmente de los superiores, o de los padres nuevos o que no entran bien en la lengua, o costumbres de Japón, antes se tengan por más aptos para esa empresa los que se aplican a confesar y ayudar las almas por los medios conformes a nuestro Instituto, y que hacen más fuerza en lo guardar e introducir que no los que ponen más conato en los catangues de Japón, aunque uno y otro se ha de procurar. Y en particular que los consultores avisen cada año si han oído a los superiores murmurar de los inferiores fuera de consulta para tratar del remedio ef. [?] guárdense todos, así superiores como inferiores de no tomar por Regla en Japón, lo que a los Japoneses más contenta, o lo que nos es más cómodo, si contradice a la sinceridad y puridad de nuestro Instituto, procurando de los hacer capaces, porque según se entiende por varias relaciones, no hay dificultad en introducir lo que quisiere más en Japón, como los tratemos cómodamente, y no se les toque en cosa de estado o hacer esclavos, o hacer tornar a la primera mujer, por do no parece conveniente comunicar con ellos si nuestro Instituto será bien recibido, ni tampoco nuestra santa ley en lo que obliga a publicarles, sino introducirlo y allanarles las dificultades que tuvieren.

- Que ningún padre azote ni de palmatoriadas ${ }^{21}$ a dogicos ni a mozos por si mismo, sino por un hermano de buena edad y virtuoso, o por un bonzo anciano.

Memorial di algunas cosas que N. P. Claudio Aquaviva ordenó para Japón que llenó el padre Matta, cuya es la mano de este mismo Memorial.

21 "Palmatorias". 


\section{Referencias}

Archivo Romano de la Compañía de Jesús (ARSJ). Roma: Congregazioni, 46, ff. 401-402, 485-488.

Arimichi, E. (1971). The meeting of cultures. En Michael Cooper (ed.), The Southern barbarians: the first Europeans in Japan. Tokyo y Palo Alto: Kodansha International in cooperation with Sophia University.

Arzubialde, S; Corella, J. y García Lomas, J.M. (1993). Constituciones de la Compañía de Jesús, Introducción y notas para su lectura. Bilbao: Ediciones Mensajero.

Covarrubias, S. O. de. (1611). Tesoro de la lengua castellana, o española compuesto por Sebastian de Cobarrubias Orozco. Madrid: Luis Sánchez.

Esquivel, J. (O.P.). (1630). Vocabulario de Japon declarado primero en portugues por los Padres de la Compañia de Iesus de aquel reyno, y agora en Castellano en el Colegio de Santo Thomas de Manila. Manila: Colegio de Santo Tomás.

Fróis, L. (1984). Historia de Japam. Edição anotada por José Wicki, S.J., Ministerio da Cultura e Coordenaçaõ Científica, Secretaria de Estado da Cultura. Lisboa: Biblioteca Nacional de Lisboa.

García Gutiérrez, F. (2012). Alessandro Valignano, S.J.: introducción de la cultura y el arte de occidente en Japón. En José Martínez Millán, Henar Pizarro Llorente, Esther Jiménez Pablo (coord.), Los jesuitas: religión, política y educación (siglos XVI-XVIII) (pp. 1471-1482). Madrid: Universidad Pontificia Comillas.

Gómez Díez, J. F. (2007). Unidad y variedad en la misión jesuítica del siglo XVI: del Japón a las fronteras de la monarquía hispánica. Estudios eclesiásticos, 321, 359-387. Madrid: Universidad Pontificia Comillas.

Lisón Tolosana, C. (2005). La fascinación de la diferencia. La adaptación de los jesuitas al Japón de los samuráis, 1549-1592. Madrid: Akal Ediciones.

López Gay, J. S. I. (1964). El matrimonio de los japoneses. Studia Missionalia, Documenta et Opera vol. 1. Roma: Libreria dell'Università Gregoriana.

López Gay, J. S. I. (1986). La inculturación de la Iglesia en Japón según el P. Alexandro Valignano, S.J. Oriente-Occidente, 1/2 (pp. 55-94). Buenos Aires: Universidad del Salvador.

O’Neill, C. E. y Domínguez, J. Ma. (2001). Diccionario histórico de la Compañía de Jesús. Biográfico-Temático, Vol. II. Madrid: Universidad Pontifica Comillas.

Romo Alonso, J. E. (2011). Jesuitas luso-castellanos en Japón (1549-1640): algunos casos paradigmáticos. En: Fernando Cid Lucas (coord.), Japón y la Península Ibérica: Cinco siglos de encuentros (pp. 359-387). Gijón: Satori. Valignano, A. (1944). Historia del principio y progresso de la Compañía de 
Jesús en las Indias orientales (1542-64). Edición de I. Wicki. Roma: Institutum Historicum Societatis Iesu.

Valignano, A. (1946). Il Cerimoniale per i missionari del Giappone, "Advertimentos e avisos acerca dos costumes e catangues de Jappão" di Alexandro Valignano,... Importante documento circa i metodi di adattamento nella Missione giapponese del secolo XVI. Testo portoghese del manoscritto originale, versione letterale italiana... Edizione critica, introduzione e note di Giuseppe Fr. Schütte,... [Presentazione di Mgr. Celso Costantini.]. Roma: Edizioni di Storia e Letteratura.

Valignano, A. (1954). Sumario de las cosas de Japón, 1583. Adiciones del sumario de Japón, 1590. Tokio: Sophia University. 